\title{
A genome-wide association study uncovers a critical role of the RsPAP2 gene in red-skinned Raphanus sativus $\mathrm{L}$.
}

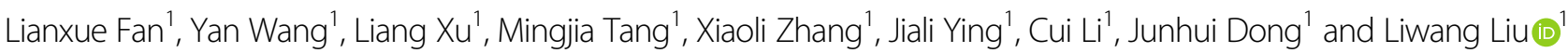

\begin{abstract}
Radish (Raphanus sativus L.) taproot contains high concentrations of flavonoids, including anthocyanins (ATCs), in redskinned genotypes. However, little information on the genetic regulation of ATC biosynthesis in radish is available. A genome-wide association study of radish red skin color was conducted using whole-genome sequencing data derived from 179 radish genotypes. The R2R3-MYB transcription factor production of anthocyanin pigment 2 (PAP2) gene was found in the region associated with a leading SNP located on chromosome 2 . The amino acid sequence encoded by the RSPAP2 gene was different from those of the other published RSMYB genes responsible for the red skin color of radish. The overexpression of the RSPAP2 gene resulted in ATC accumulation in Arabidopsis and radish, which was accompanied by the upregulation of several ATC-related structural genes. RsPAP2 was found to bind the RsUFGT and RsTT8 promoters, as shown by a dual-luciferase reporter system and a yeast one-hybrid assay. The promoter activities of the RSANS, RSCHI, RSPAL, and RSUFGT genes could be strongly activated by coinfiltration with RSPAP2 and RsTT8. These findings showed the effectiveness of GWAS in identifying candidate genes in radish and demonstrated that RsPAP2 could (either directly or together with its cofactor RsTT8) regulate the transcript levels of ATC-related genes to promote ATC biosynthesis, facilitating the genetic enhancement of ATC contents and other related traits in radish.
\end{abstract}

\section{Introduction}

Radish (Raphanus sativus L.) taproot is a valuable source of phytochemicals such as flavonoids. Anthocyanins (ATCs) are a subgroup of flavonoids that exhibit strong antioxidant activity that is beneficial for human health ${ }^{1}$. Previous studies have reported that the major components of ATC pigments in red radish epidermal tissue are acylated pelargonidin glycosides with a combination of p-coumaric, ferulic, or malonic acids ${ }^{2,3}$. Pelargonidin exhibits an orange-red hue, and acylation is expected to shift the hue to red and improve stability ${ }^{2}$, leading to the formation of red skin in radish.

ATCs are synthesized via the flavonoid branch of the general phenylpropanoid pathway ${ }^{4}$. The initial steps of

\footnotetext{
Correspondence: Liwang Liu (nauliulw@njau.edu.cn)

${ }^{1}$ National Key Laboratory of Crop Genetics and Germplasm Enhancement, Key Laboratory of Horticultural Crop Biology and Genetic Improvement (East China) of MOAR, College of Horticulture, Nanjing Agricultural University, 210095 Nanjing, PR China
}

the phenylpropanoid pathway involve phenylalanine ammonia lyase (PAL), cinnamate 4-hydroxylase $(\mathrm{C} 4 \mathrm{H})$, and 4-coumarate-CoA ligase (4CL). Subsequently, the flavonoid pathway is initiated by the condensation of one molecule of 4-coumaroyl-CoA with three molecules of malonyl-CoA by chalcone synthase (CHS). The successive steps catalyzed by chalcone isomerase $(\mathrm{CHI})$, flavanone 3-hydroxylase (F3H), dihydroflavonol-4-reductase (DFR), anthocyanidin synthase (ANS), and flavonoid 3oglucosyltransferase (UFGT) lead to the production of ATC pigments ${ }^{5,6}$. These crucial structural genes in the ATC biosynthesis pathway have been isolated and well characterized in plant species such as Arabidopsis, apple, eggplant, and grape ${ }^{1,7-10}$.

ATC biosynthesis is known to be transcriptionally regulated by R2R3-MYB transcription factors (TFs), basic helix-loop-helix (bHLH) TFs and WD40 repeat proteins, which normally form MYB-bHLH-WD40 complexes $^{1,8}$. The conserved complex interacts with a 
7-bp MYB-recognizing element (MRE, "ANCNNCC") and a 6-bp bHLH-recognizing element (BRE, "CACN[A/ $\mathrm{C} / \mathrm{T}][\mathrm{G} / \mathrm{T}]$ ") in the promoter regions of ATC structural genes in various plant species ${ }^{11}$. Among these regulators, MYB TFs are considered essential regulators of ATC production; these TFs include MYB75 (production of anthocyanin pigment 1, PAP1), MYB90 (PAP2), MYB113 and MYB114 in Arabidopsis ${ }^{12-14}$, MdMYB10,

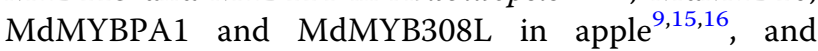
PyMYB10 and PyMYB114 in Chinese pear ${ }^{17,18}$. In addition, a few members of the bHLH TF family, such as AtTT8 (bHLH42) in Arabidopsis and StbHLH1 in potato, also result in enhanced activity at promoters containing a cis-regulatory element that is conserved in several ATC biosynthetic genes ${ }^{19,20}$. Most of the structural genes known to be involved in ATC biosynthesis have been reported in radish ${ }^{21}$; however, only a few TFs have been clearly identified as being related to the transcriptional regulation of the ATC biosynthesis pathway in radish, such as RsMYB1, RsMYB1a, RsMYB90, and RsTT8 ${ }^{22-27}$.

Genome-wide association studies (GWASs) are a valuable, powerful tool for dissecting the genetic basis of complex traits in plants ${ }^{28,29}$. The GWA mapping of 360 natural genotypes of Arabidopsis revealed that MYB90 was the major gene responsible for the wide variation in ATC accumulation ${ }^{14}$. A total of six candidate loci responsible for ATC variation in lettuce leaves were identified using a GWAS approach ${ }^{29}$. The peach $F 3 H$ gene was found in the associated region of a GWAS signal for flesh color $^{30}$. Moreover, GWAS has been applied to investigate ATC pigmentation and fruit color in eggplant ${ }^{31}$ grape $^{32}$, and rose ${ }^{33}$. These studies indicate the usefulness of GWAS for deciphering the genetic control of ATC biosynthesis and red skin color in radish taproots.

In this study, to identify key ATC-specific structural genes or regulatory factors and better understand the genetic regulation of red skin color in radish, a GWAS for radish red skin color was conducted in a diverse population of 179 radish genotypes. An R2R3-MYB TF, RsPAP2, was identified as being associated with ATC biosynthesis in red-skinned radish taproot using highquality single nucleotide polymorphisms (SNPs). The overexpression of the RsPAP2 gene in Arabidopsis plants and radish cotyledons positively promoted red pigmentation. RsPAP2 could bind the promoters of several ATC biosynthetic genes, either directly or with its partner RsTT8, to promote ATC biosynthesis, indicating that the RsPAP2 gene is a critical transcriptional activator for ATC production in radish. This study provides valuable information for the clarification of the molecular mechanism underlying taproot skin coloration and will facilitate the genetic improvement of ATC-associated traits in radish breeding programs.

\section{Results \\ SNP genotyping, population structure, and linkage disequilibrium (LD) analyses}

A total of 179 cultivated radish genotypes were selected for whole-genome resequencing analysis (Table S1), generating $819.71 \mathrm{~Gb}$ of clean reads $(\sim 4.58 \mathrm{~Gb}$ for each sample on average). The clean reads from each genotype were mapped to the "WK10039" radish genome ${ }^{34}$. The mapping rate varied from 70.48 to $94.73 \%$ among different genotypes, with an average of $89.70 \%$ (Table S1). A total of $1,222,458$ high-quality SNPs were detected, among which 588,896 SNPs (48.17\%) were located within genes (Fig. S1a). The SNP density was consistent with the density of genes on the nine radish chromosomes (Fig. S2). Moreover, a total of 98,730 SNPs were suggested to have a potential impact on gene function as splicing, stop-gain, stop-loss, and nonsynonymous variants (Fig. S1b).

Based on the SNP data, a neighbor-joining tree was constructed to show the phylogenetic relationships among 179 cultivated radish genotypes (Fig. 1a). The "NAU-145" and "NAU-175" genotypes, with black skin, were clustered into one clade (Cluster I), while the remaining 177 genotypes from Asia were divided into four other clusters. Cluster II contained 31 long-white genotypes and the red-skinned genotype "NAU-017". Fifty redskinned genotypes and three landraces ("NAU-041", "NAU-083", and "NAU-155") were classified into Cluster III. Cluster IV included 59 landraces from China, while Cluster V consisted of 5 genotypes from Japan ("NAU116", "NAU-124", "NAU-133", "NAU-136", and "NAU140 ") and 28 other accessions. A structure analysis showed the minimum cross-validation error at $K=5$, which was determined to be the optimum $K$-value for the radish population (Fig. 1b). Principal component analysis (PCA) was performed, and PC1 and PC2 differentiated five radish clusters (Fig. 1c), which was supported by both phylogenetic and structural analyses. In addition, the PCA plot indicated that the long-white genotype from Cluster II was more distantly related to the other genotypes, especially the red-skinned genotype from Cluster III, consistent with their phenotypic variations in skin color.

The LD level was measured as the physical distance at which $r^{2}$ decreased to 0.20 , and LD decay was estimated to be $7 \mathrm{~kb}$ when all genotypes were analyzed. LD decay in the five clusters varied from 2.5 to $22.8 \mathrm{~kb}$, with the largest $(22.8 \mathrm{~kb})$ decay observed for the genotypes with black skin (Fig. 1d).

\section{GWAS analysis for red skin color in radish taproots}

Considering the phylogenetic relationships and the variations in skin color among the 179 radish genotypes, 65 white- and 95 red-skinned genotypes were singled out 


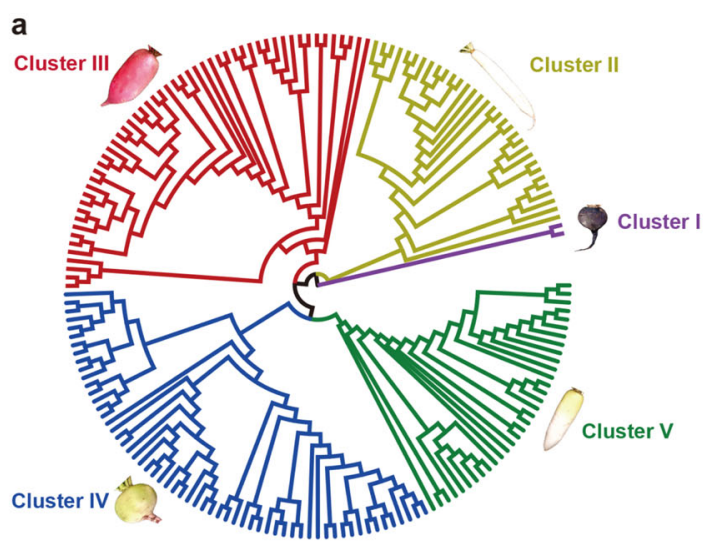

b

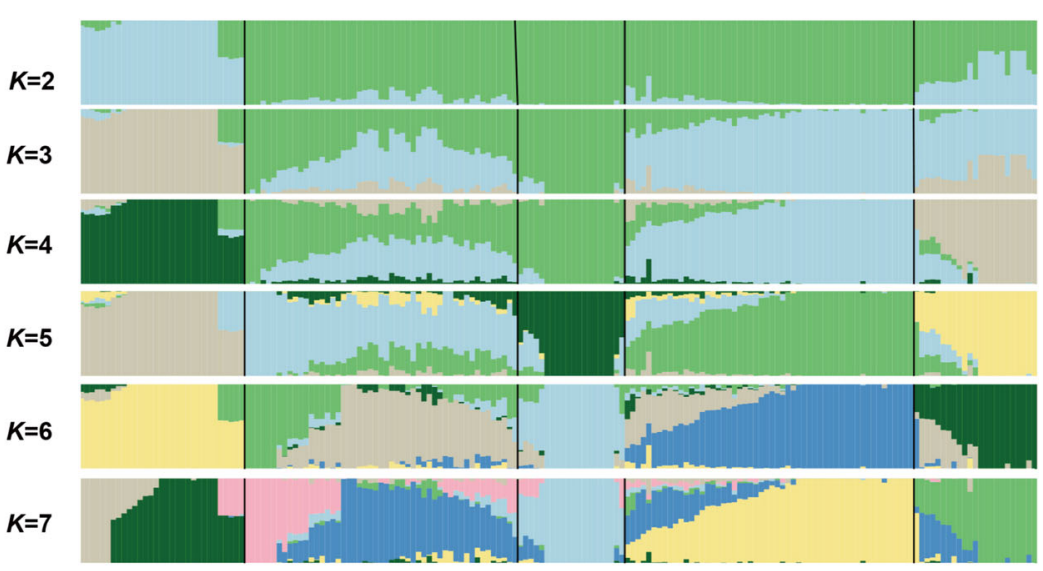

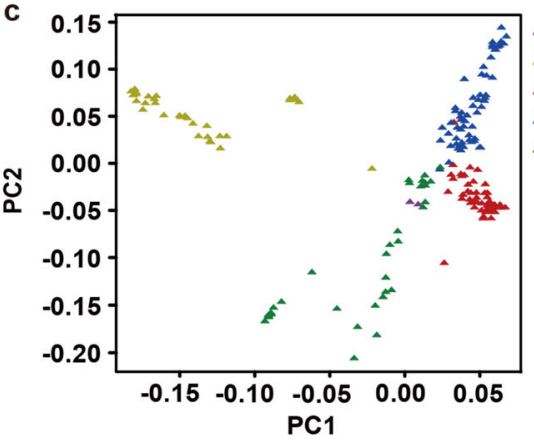

d

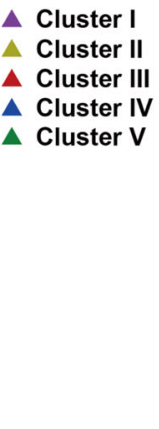

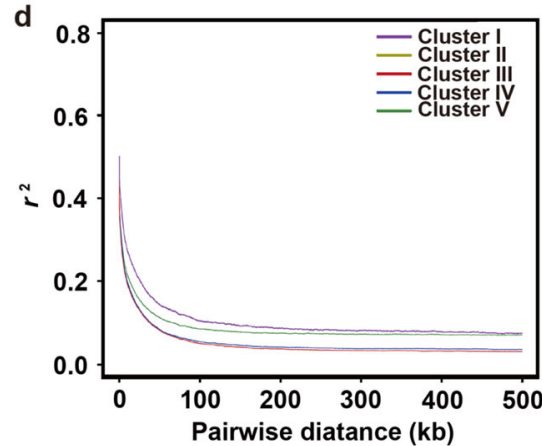

Fig. 1 Population structure of radish genotypes. a Neighbor-joining phylogenetic tree. $\mathbf{b}$ Population structure analysis with different numbers of clusters $(K=2-7)$. c PCA of all radish genotypes. The first two principal components (PC1 and PC2) were used to visualize the relationships among individuals and groups. Each point represents an independent radish genotype. $\mathbf{d}$ Decay of LD in five clusters measured by $r^{2}$

for the association analysis of red skin color (Fig. 2a). A total of 14 significantly associated SNP signals containing 26 SNPs were detected at the applied significance threshold $\left(-\log _{10} P>7\right)$. Notably, two leading SNPs were observed on chromosomes (Chrs) 1 and 2 (Fig. 2b and Table S2), which were located in an unknown gene (Rs033130) and a calmodulin-binding transcription activator (Rs095780), respectively. However, both of these SNPs were synonymous variation.
Based on the LD level, a total of 39 candidate genes were identified in the space defined by the $\pm 10 \mathrm{~kb}$ regions on either side of the 14 peak SNPs, such as the production of anthocyanin pigment 2 (PAP2, Rs095840), Myb domain protein 92 (Rs144280), and zinc finger protein-related (Rs144260) genes (Table S2). Remarkably, RsPAP2, located on Chr2, was associated with one of the top two SNPs (Fig. 2c), indicating that it might be a potential candidate responsible for the red skin color of radish. 
a

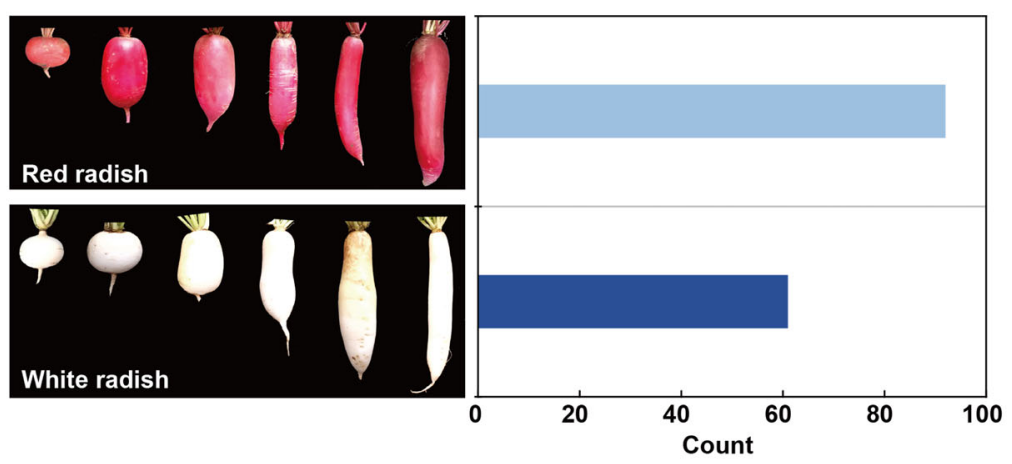

b
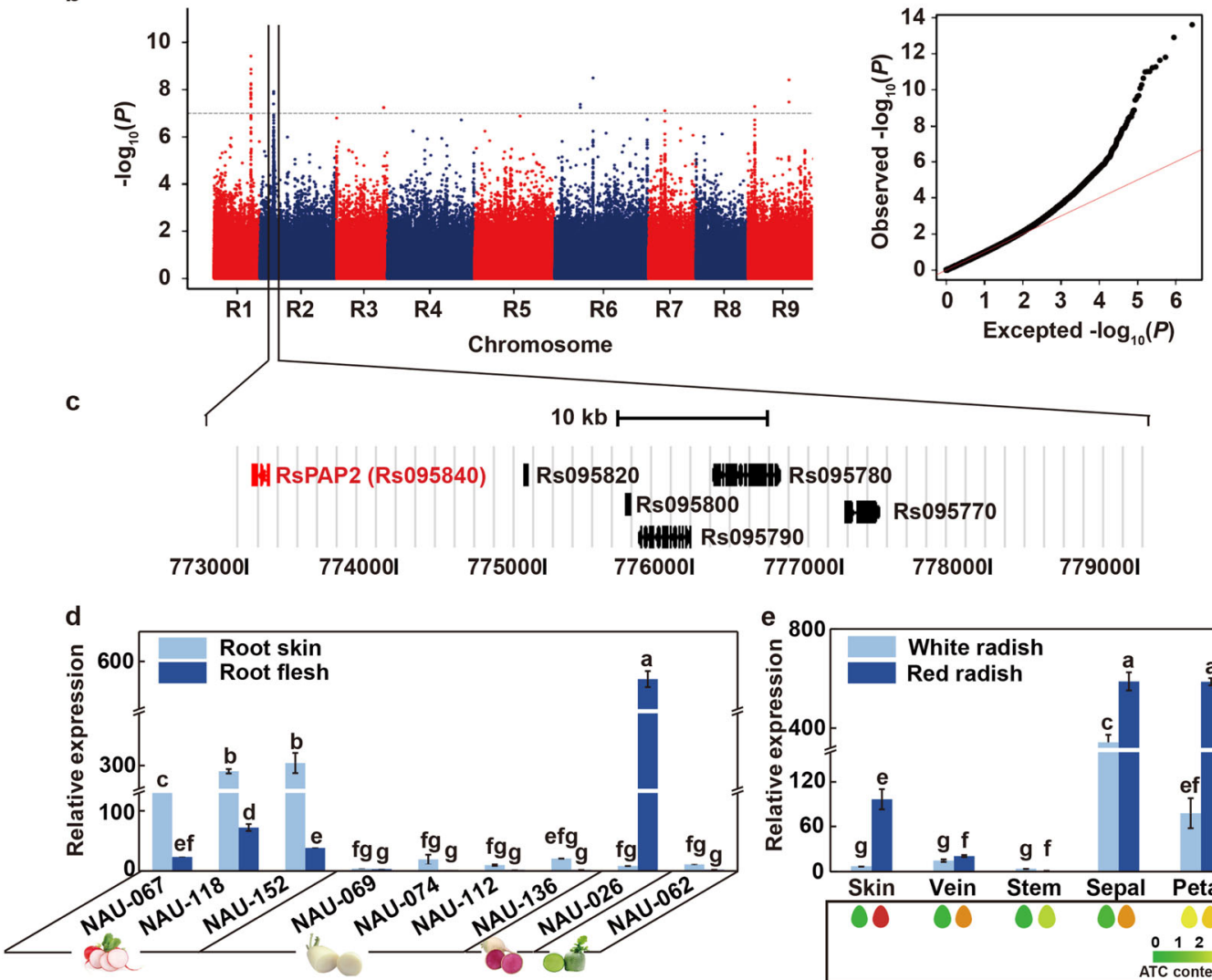

095780

Rs095770

$7780001 \quad 7790001$

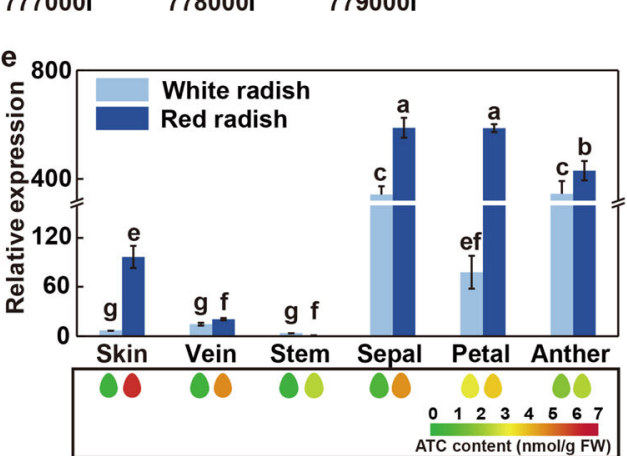

Fig. 2 GWAS analysis for red skin color in radish. a Frequency distribution of skin color (red and white) variations across 179 radish genotypes. b Manhattan plots (left) and quantile-quantile plots (right) depicting results of the GWAS for red skin color. The $x$-axis depicts the physical location of SNPs across the 9 chromosomes of radish, and the $y$-axis depicts the $-\log _{10}(P$ value). $\mathbf{c}$ All genes positioned in the region around the leading SNPs on Chr2. d e Relative expression patterns of the candidate gene RSPAP2 in the roots of nine genotypes and in different tissues of the white- and redskinned genotypes ("NAU-069" and "NAU-067"), respectively. Heatmap showing the total ATC content of different tissues from "NAU-069" and "NAU067", respectively. Error bars indicate the standard deviation (SD) from at least three replicates. Letters represent significant differences at the 0.05 level based on Duncan's test

Identification of the candidate gene RsPAP2 responsible for red skin color

The PAP2 gene, also known as the "MYB90 TF", is responsible for determining ATC contents in Arabidopsis and rose petals ${ }^{14,33}$. A MYB90 TF, RsPAP2, was also detected in the associated region of the leading SNP located on Chr2 (Fig. 2b, c and Table S2). The 720-bp coding sequence of the RsPAP2 gene was isolated from the red-skinned radish genotype "NAU-067", encoding a polypeptide of 239 amino acids. In the phylogenetic analysis of ATC-related R2R3-MYB proteins from radish and Arabidopsis, RsPAP2 from "NAU-067" was clustered into a clade together with the published radish MYBs and AtMYB75/PAP1, AtMYB90/PAP2, and AtMYB113 from Arabidopsis (Fig. S3a). The published radish MYBs were further divided into four clades (RsMYB I, RsMYB II, 
RsMYB III, and RsMYB IV), but RsPAP2 did not belong to any of these clades (Fig. S3a), indicating that they were different genes. In particular, RsPAP2 and RsMYB1.1 (CX16Q-25-2), located on Chr2, showed significant differences between their amino acid sequences (Fig. S4). Moreover, the amino acid sequence of RsMYB1.1 was subjected to BLAST searches against the radish genome of WK10039, and it was found that RsMYB1.1 was located at the position of Rs094840 (Chr2: 8,425,153-8,426, $787 \mathrm{bp}$ ), which is different from that of the RsPAP2 gene (Rs095840, Chr2: 7,730,000-7,731,000 bp) (Fig. S3b). In addition, the RsPAP2 protein contained the complete R2R3 domain at the amino terminus. A bHLH motif ([D/ $\left.E] \mathrm{Lx}_{2}[\mathrm{R} / \mathrm{K}] \mathrm{x}_{3} \mathrm{Lx}_{6} \mathrm{Lx} x_{3} \mathrm{R}\right)$ was found in the $\mathrm{R} 3$ domain (Fig. S4), suggesting that RsPAP2 belonged to an R2R3MYB family member. In addition, a total of three nonsynonymous SNPs were identified in the coding region of RsPAP2 among five red-skinned and five white-skinned radish genotypes, which resulted in three amino acid substitutions in the protein sequence of RsPAP2 (Fig. S5). However, two substitutions were only identified in "NAU009", "NAU-090", and "NAU-103", while the other substitution was not located in a conserved domain (Fig. S5). Therefore, these substitutions are unlikely to affect protein activity.

\section{The expression pattern of RsPAP2 is consistent with ATC content in radish}

The transcript abundance of the RsPAP2 gene in nine radish genotypes with different skin and flesh colors was measured by real-time quantitative polymerase chain reaction (RT-qPCR) analysis (Fig. 2d). The results showed that the transcriptional levels of RsPAP2 in root skin were 6.5-, 4.0-, and 8.0-fold higher than those in root flesh among the three red-skinned genotypes "NAU-067", "NAU-118", and "NAU-152", respectively (Fig. 2d). In the genotype "NAU-026" with green skin and red flesh, RsPAP2 was significantly upregulated in root flesh compared with root skin (Fig. 2d). However, the expression levels of RsPAP2 in four genotypes with white skin and flesh ("NAU-069", "NAU-074", "NAU-112", and "NAU136") and one radish with green skin and flesh ("NAU062") exhibited no significant difference in the skin or flesh (Fig. 2d). Furthermore, the RsPAP2 gene exhibited high expression levels in tissues with abundant ATC accumulation, such as the root skin, leaf veins, stem, sepals, petals, and anthers of the red-skinned genotypes (Fig. 2e). These results indicated that the RsPAP2 gene is an important regulator of the red skin color of radish taproots.

\section{Stable expression of RsPAP2 promotes ATC biosynthesis in Arabidopsis}

To verify its potential function in ATC biosynthesis, an overexpression vector containing RsPAP2 driven by the
CaMV35S promoter was transformed into wild-type Arabidopsis plants (WTs) (Fig. S6). Among the 19 transgenic RsPAP2-overexpressing Arabidopsis lines (RsPAP2-OEs), eight displayed different levels of purple coloration on the leaves, roots, and stems near inflorescences, but no purple or red coloration was observed in other organs (Fig. 3a). Consistent with the expression levels of the RsPAP2 transgene, the total ATC contents in the roots and leaves of RsPAP2-OEs were $\sim 25.9$ - and 47.8fold higher than those in the WTs, respectively (Fig. 3b). These results indicated that the heterologous expression of RsPAP2 promoted ATC accumulation in the roots and leaves of Arabidopsis.

To further investigate the molecular basis of the reddish phenotypes of the different tissues in the transgenic lines, 13 key endogenous genes, including nine structural and four regulatory genes related to ATC biosynthesis, were analyzed by RT-qPCR in the roots and leaves of RsPAP2$\mathrm{OE}$ plants (Fig. 3b). The results showed that $A t C 4 H$, AtCHS, AtANS, and AtTT8 were significantly upregulated in both the roots and leaves of the RsPAP2-OEs compared with the WTs, whereas AtDFR was downregulated. AtPAL1 and AtCHI were specifically upregulated in the roots and leaves, respectively. Interestingly, four other ATC-related genes, AtF3H, AtUFGT, AtPAP2, and $A t M Y B 113$, exhibited upregulated expression in the leaves of the RsPAP2-OE plants but showed the opposite expression pattern in the roots. These findings indicated that RsPAP2 could activate the ATC biosynthesis pathway at the transcriptional level in Arabidopsis plants.

\section{RsPAP2 induces ATC biosynthesis in radish cotyledons by promoting the expression of ATC-related genes}

The transient transformation of radish with RsPAP2 was further performed to investigate its role in ATC production. Some pigmentation was observed after transformation with RsPAP2 at the infiltration sites in radish cotyledons (Fig. 4a). Consistent with the visual phenotype, the total ATC contents of the reddish cotyledons were 4.3- and 10.1-fold higher than those in the control transformed with the empty vector. As expected, the expression levels of the RsPAP2 gene in the infiltrated cotyledons were 1.3 and 2.3 times higher than those in the control (Fig. 4b). To determine how the radish cotyledons produce red pigments, eight ACT-related genes were selected for RT-qPCR analysis (Fig. 4b). Six structural genes (RsPAL, RsC4H, Rs4CL, RsCHI, RsF3H, and RsUFGT) were significantly upregulated in the reddish cotyledons compared with the control. In addition, the expression of RsTT8, a regulator of ATC biosynthesis, was upregulated in cotyledons overexpressing RsPAP2 (Fig. 4b), demonstrating that RsPAP2 induces ATC production via the transcriptional activation of ATC biosynthetic genes in radish. 
a
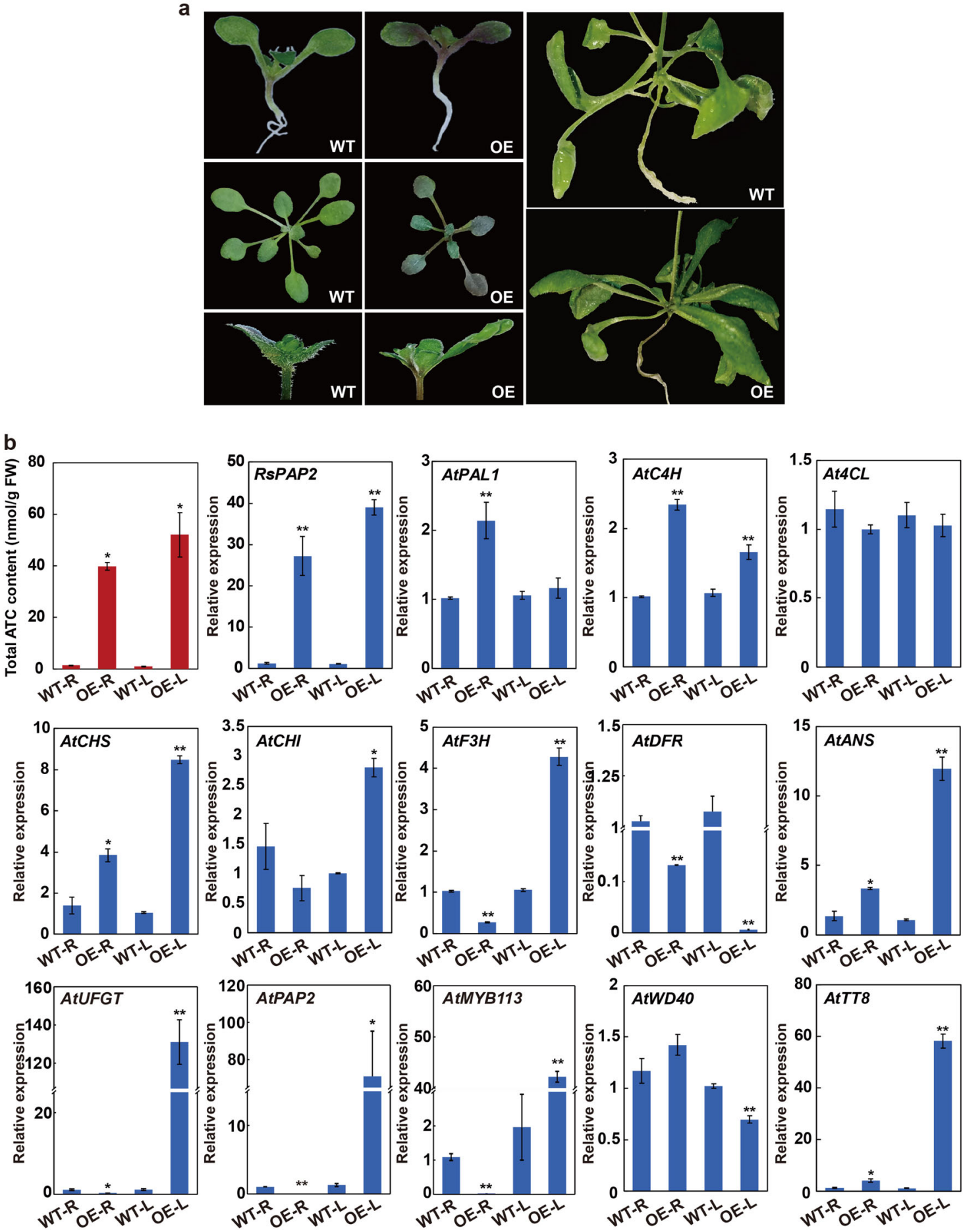

Fig. 3 Functional analysis of RsPAP2 in Arabidopsis. a Phenotype of RsPAP2-overexpressing Arabidopsis plants. The 15-day-old leaves, 30-day-old leaves, roots, and the stems near the inflorescences of the transgenic plants were purple. $\mathbf{b}$ Total ATC contents (top-left) and relative expression levels of RSPAP2 and 13 endogenous ATC-related genes in the roots (R) and leaves (L) of 30-day-old transgenic Arabidopsis. WT wild-type Arabidopsis plants, OE RSPAP2-overexpressing Arabidopsis plants. The error bar represents the SD from three biological replicates in three parallel experiments, and statistical significance was determined using Student's $t$ test. ${ }^{*} p<0.05 ;{ }^{* *} p<0.01$ 
a
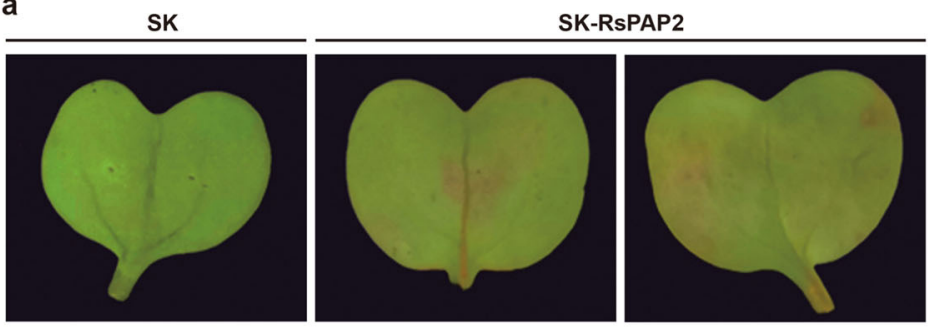

b
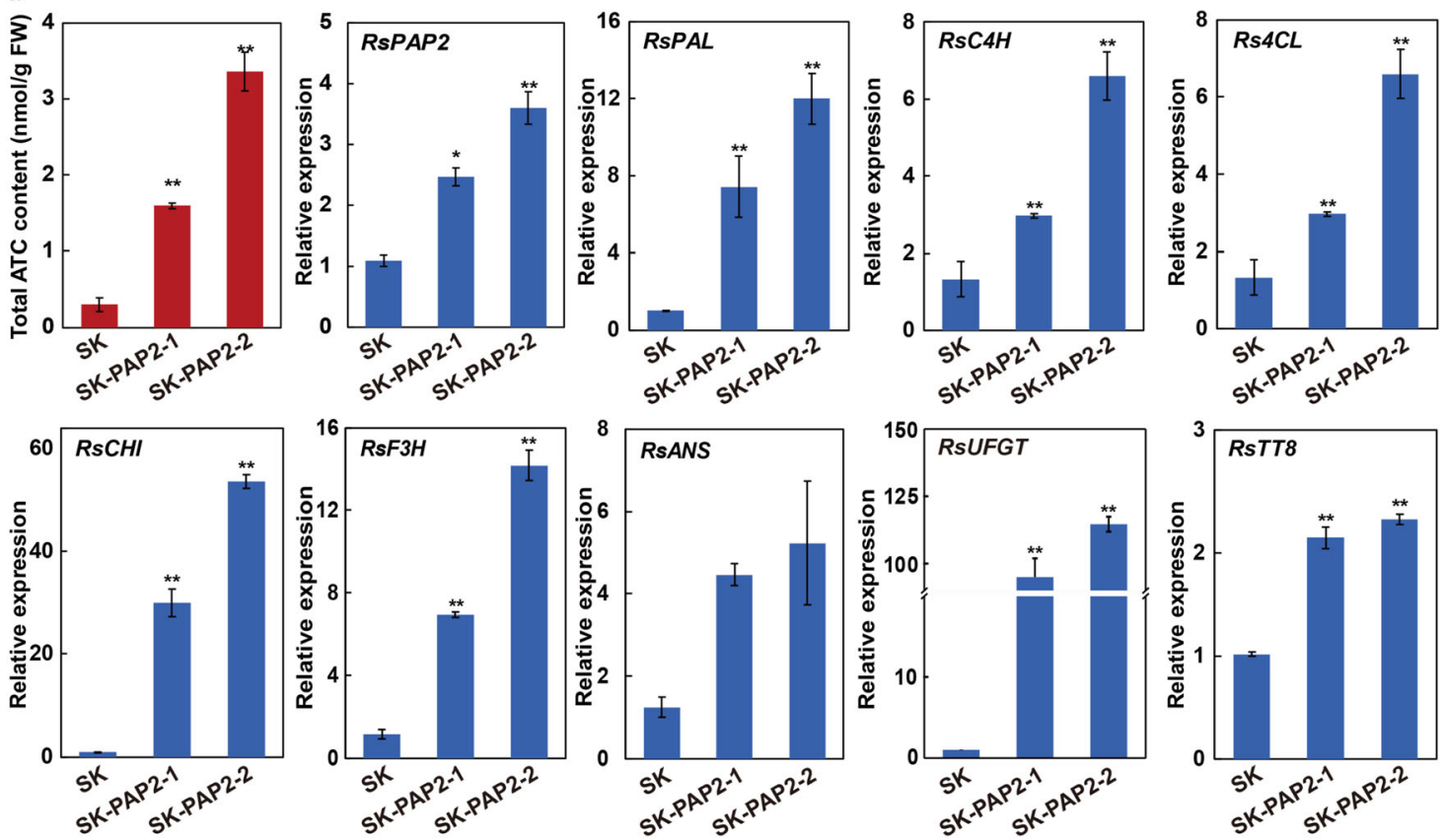

Fig. 4 Transient transformation analysis of radish cotyledons. a Radish cotyledon transiently expressing the empty vector (SK) and RsPAP2 (SKRSPAP2). b Total ATC contents (top-left) and relative expression levels of RSPAP2 and eight ATC-related genes in reddish cotyledons. Error bars represent the SD from three biological replicates in three parallel experiments assessed by RT-qPCR. Statistical significance was determined using Student's $t$ test. ${ }^{*} p<0.05 ;{ }^{* *} p<0.01$

To examine the ability of RsPAP2 to transactivate the promoters of ATC biosynthetic genes, transient dualluciferase assays in tobacco leaves were carried out using the constructs shown in Fig. 5a. When RsPAP2 was transfected alone, the activities of two segmented promoters of the RsUFGT ( $\left.P_{\text {RsUFGT-477 }}\right)$ and RsTT8 $\left(P_{\text {RsTT8- }}\right.$ $\left.{ }_{640}\right)$ genes were significantly increased by 47.0 - and $22.9-$ fold, respectively (Fig. 5b). In a yeast one-hybrid $(\mathrm{Y} 1 \mathrm{H})$ system (Fig. 5c), RsPAP2 was further confirmed to directly bind the promoters of RsUFGT (P $\left.P_{\text {RsUFGT-477 }}\right)$ and RsTT8 $\left(P_{\text {RsTT8-640) }}\right.$ (Fig. 5d).

In addition, stronger activation of $P_{R s A N S-501}, P_{R S C H I-682}$, $P_{R s P A L 665}, P_{R s U F G T-285}$, and $P_{R s U F G T-477}$ was detected when tobacco leaves were simultaneously cotransformed with RsPAP2 and RsTT8 (Fig. 5b), indicating that the RsPAP2-RsTT8 complex might exhibit a broader transcriptional regulatory ability for ATC-related genes. These results indicated that RsPAP2 is an important regulator that selectively induces the expression of several ATC biosynthetic genes to promote ATC accumulation in radish.

\section{Discussion}

Radish taproots provide an excellent source of valuable nutrients, including dietary fiber, sugar, vitamins, and phytochemicals ${ }^{35,36}$. ATCs are responsible for the red skin and flesh color of radish taproots and exhibit nutritional and pharmaceutical properties ${ }^{22,37}$. In a previous study, several genes regulating skin or flesh color in radish taproots have been reported. Based on homology, RsMYB1 and RsMYB1a were separately isolated from the red radish cultivars "Bordeaux" and "Hongxin 1", and their positive regulation of ATC production was confirmed $^{22,25}$. Transposon-induced methylation of the RsMYB1 promoter was reported to disturb ATC accumulation in red-fleshed radish ${ }^{27}$. The RsMyb1/RsMYB90 
a

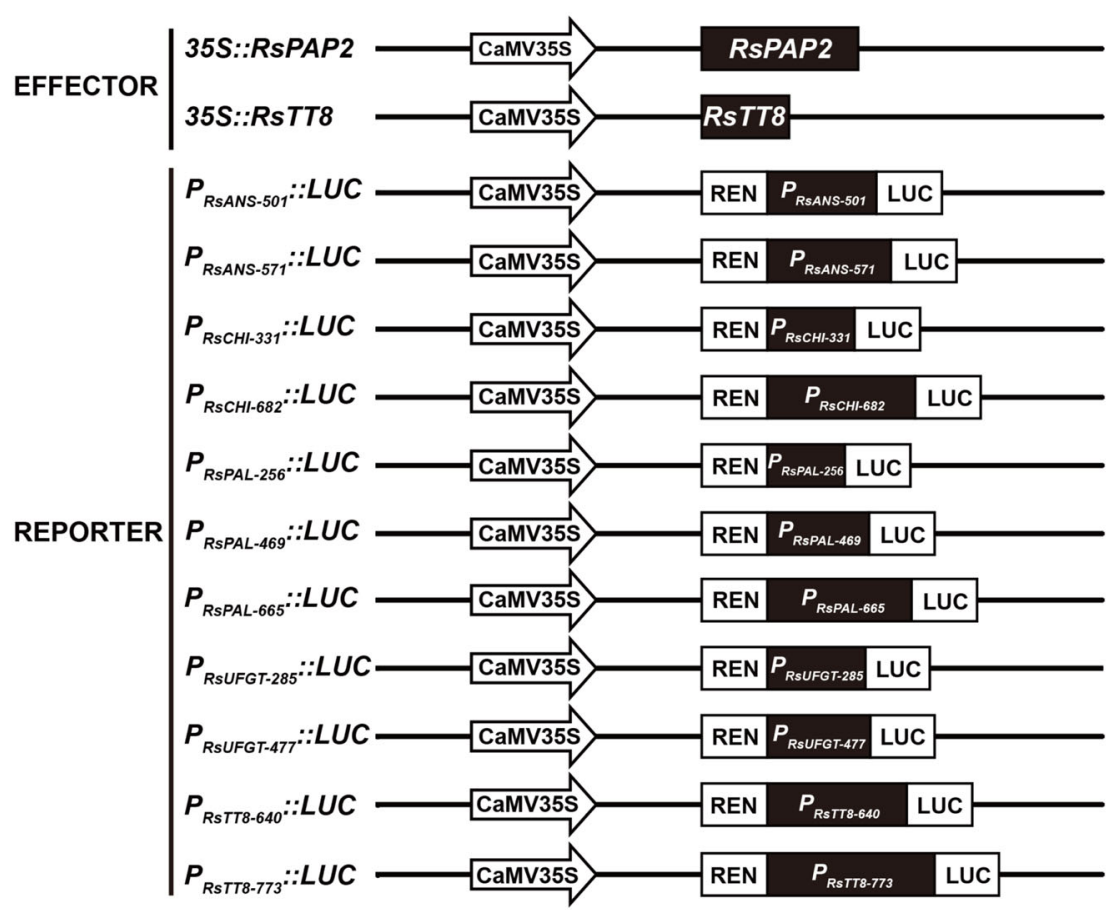

b

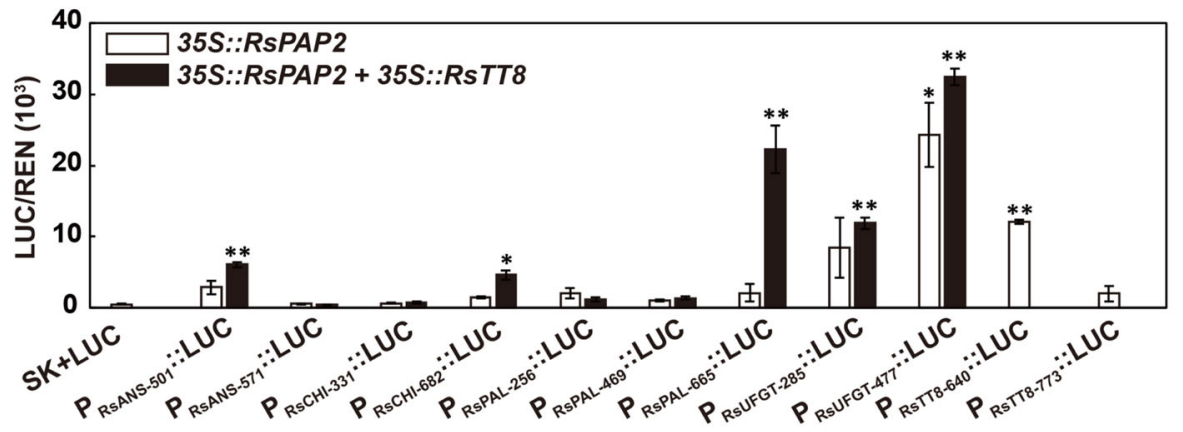

C

d

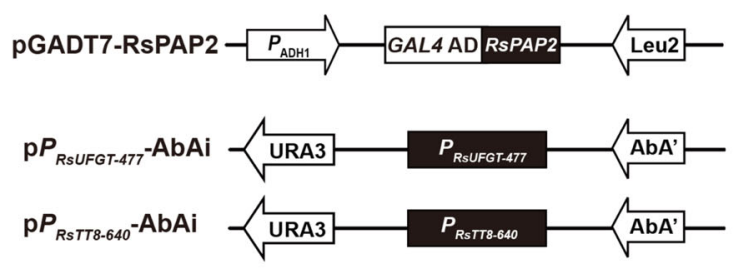

SD/-Leu SD/-Leu+AbA

SD/-Leu SD/-Leu+AbA

$P_{\text {RSUFGT-477 }}$
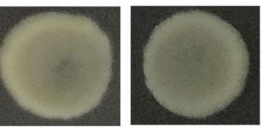

$\boldsymbol{P}_{\text {RsTT8-640 }}$

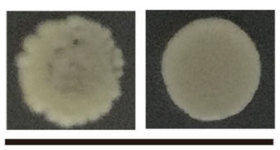

pGADT7-RsPAP2

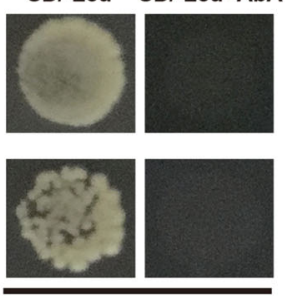

pGADT7

Fig. $\mathbf{5}$ In vivo and vitro interactions of TFs and ATC-related structural gene promoters. a Construct details for dual-luciferase assays. $\mathbf{b}$ The in vivo associations of TFs (RSPAP2 and RsTT8) and promoters were obtained from dual-luciferase assays in tobacco leaves. The REN and LUC activities

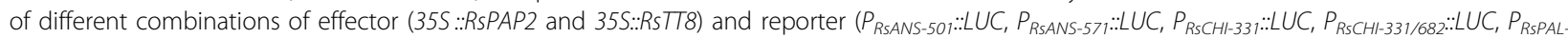

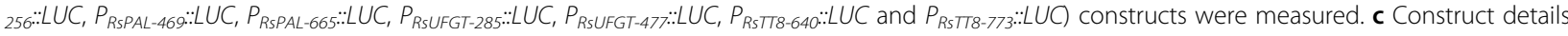

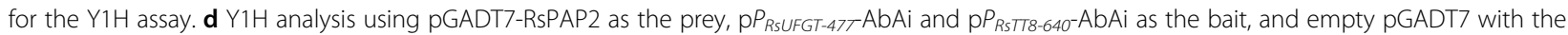
corresponding recombinant PABAi as a negative control. Error bars represent the SD from three biological replicates in three parallel experiments. Statistical significance was determined using Student's $t$ test. ${ }^{*} p<0.05 ;{ }^{*} p<0.01$

gene is a crucial regulator of radish root skin color $^{23,26}$. Liu et al. reported that the RsMYB1.1 gene was located within the mapping region for the purple skin of radish $\operatorname{root}^{24}$. These findings suggested that MYB TFs are the crucial determinants of ATC accumulation in red-skinned radish and that $M Y B$ genes from different plant materials 
might control skin color inheritance via different mechanisms. Therefore, the identification of major genes controlling red skin color across diverse radish genotypes is necessary, which will contribute to revealing the genetic regulation of skin color in radish fleshy roots. The GWAS method plays important roles in the identification of candidate genes related to ATC content in some models and important crop species ${ }^{14,30,33}$. In this study, a GWAS strategy was first applied based on whole-genome sequencing to analyze factors influencing the red skin color of radish.

\section{GWAS analysis of genetic factors underlying the red skin color of radish}

In the present study, a natural radish population containing abundant phenotypic and genetic variations was subjected to GWAS analysis. The GWAS results indicated that there were two leading SNPs on Chr1 and Chr2 associated with red skin color, indicating that at least these two genes might control radish red skin color. Considering LD levels, the candidate gene regions became the focus of efforts to identify key genes related to red skin color $^{38}$. Remarkably, the RsPAP2 (Rs095840) gene, encoding the R2R3-MYB90 protein, was identified and located on Chr2. Phylogenetic analysis showed that RsPAP2 differed from other published MYBs responsible for skin color in radish and presented a closer relationship with AtMYB90/PAP2 in Arabidopsis (Fig. S3a). These results suggested that RsPAP2 was a novel potential R2R3-MYB gene responsible for red skin color in radish taproots.

\section{RsPAP2 TF promotes ATC biosynthesis in radish}

The RsPAP2 gene was found to exhibit high transcript levels in all ATC-rich tissues of radish and showed a significant positive correlation with ATC content. To understand the mechanism leading to differential gene expression in red- and white-skinned genotypes, the coding and promoter sequences of the RsPAP2 gene were compared among the different radish genotypes. It was found that there were three nonsynonymous SNPs in the coding region of RsPAP2, but two of the SNPs were only identified in three genotypes with different skin colors, and one SNP was not located in a conserved domain, which similar to the situation of the SlAN2-like gene in tomato fruit ${ }^{39}$. However, a total of ten SNPs and four base deletions were found in the $948 \mathrm{bp}$ promoter region, among which two of the base deletions ("T" at $849 \mathrm{bp}$ and "A" at $850 \mathrm{bp}$ ) were consistently found among the redskinned genotypes with the exception of one genotype, "NAU-123" (Table S3). Cases of such insertions or deletions are also found in blood orange, apple, and tomato $^{39-41}$. Although further experimental evidence is needed, this deletion in the promoter region might contribute to the differential expression of RsPAP2 and affect ATC biosynthesis in radish.

\section{RsPAP2, either alone or with RsTT8, regulates ATC accumulation in radish}

To further understand the positive effect of RsPAP2 on ATC production in radish, RsPAP2-overexpressing Arabidopsis lines were obtained via the floral-dip method. The stable expression of RsPAP2 driven by the CaMV35S promoter in Arabidopsis led to ATC accumulation in the roots and leaves, similar to a recent report in Oilseed rape ${ }^{42}$. In addition, the radish cotyledons showed distinct accumulation of red pigments after infiltration with RsPAP2 alone. Moreover, the transcript levels of several key structural genes exhibited significant increases in both RsPAP2-overexpressing Arabidopsis plants and radish cotyledons. This phenomenon has also been noted for genes in other plants, such as OvPAP2 in oilseed rape ${ }^{42}, N t M Y B 2$ in Chinese narcissus ${ }^{43}$, and $A g M Y B 2$ in purple celery ${ }^{44}$. These findings indicated that RsPAP2 might promote ATC biosynthesis by positively regulating several critical ATC-related genes in radish. Both dual-luciferase and $\mathrm{Y} 1 \mathrm{H}$ assays further confirmed that RsPAP2 could specifically bind to the promoters of RsUFGT and RsTT8, consistent with previous reports in pear $^{45}$ and grape ${ }^{46}$. In addition, AtUFGT and RsUFGT genes were intensively upregulated by the overexpression of the RsPAP2 gene in Arabidopsis leaves and radish cotyledons, respectively. This result suggested that the RsUFGT gene might be the major control point for ATC accumulation in radish ${ }^{21}$.

In addition, the cotransformation of RsPAP2 and RsTT8 demonstrated that the complex could activate the promoters of more structural genes, including RsPAL, RsANS, $R s C H I$, and RsUFGT, indicating that RsTT8 was a partner of RsPAP2 in the regulation of ATC biosynthesis, which might serve as a transcriptional regulatory mechanism of ATC biosynthesis in radish (Fig. 6). RsPAP2 could promote ATC accumulation by binding the MRE element in the RsUFGT promoter. It could also act in combination with its cofactor RsTT8 to regulate the activity of additional structural genes (e.g., RsPAL, RsANS, RsCHI, and RsUFGT) to promote the ATC biosynthesis pathway. Moreover, it was found that RsPAP2 could function in the transcriptional activation of RsTT8, which might be another way to positively increase ATC accumulation in radish. In addition, one SNP within a calmodulin-binding transcription activator protein (CBP) gene was detected on Chr2; this SNP was associated with red skin color, and a homolog of this gene, CBP60g, has been reported to regulate the expression of MYBs to control ATC biosynthesis in Arabidopsis ${ }^{47,48}$. A CBP binding motif was also predicted in the RsPAP2 promoter region. Therefore, it is possible that RsPAP2, activated by RsCBP, plays an 


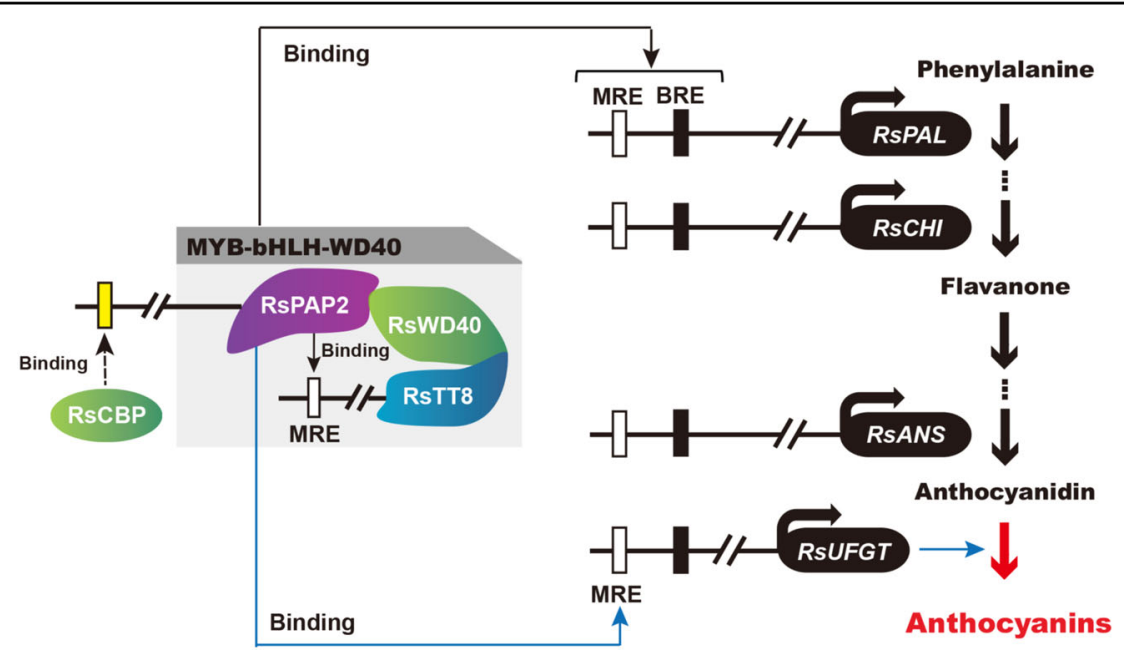

Fig. 6 Model for the transcriptional regulation of ATC biosynthesis in radish. The white, black, and yellow boxes represent the MYB-recognizing element (MRE), bHLH-recognizing element (BRE), and CBP binding motif, respectively

essential role in radish ATC accumulation, which needs to be confirmed by further research.

A previous study indicated that the genetic mechanisms determining radish root skin color differ among different genotypes $^{24}$. Although several ATC-related RsMYB genes have been identified by QTL-seq, traditional linkage analysis and genome resequencing using hybrid populations $^{23,24,26}$, the available information is far from complete to fully explain the red skin color variations among diverse radish genotypes. In the present study, to enrich the understanding of ATC biosynthesis regulation, the GWAS approach was first applied to address potential genes responsible for red skin color among various redskinned radish genotypes. An R2R3-MYB TF, RsPAP2, was identified as a candidate gene for red skin color. Lim et al. confirmed that RsMYB1 is a positive regulator of ATC accumulation in a study involving transgenic Arabidopsis and tobacco plants ${ }^{22}$. In our study, based on the observation of transgenic Arabidopsis and radish cotyledons, a hypothetical regulatory network of radish ATC biosynthesis regulated by RsPAP2 was proposed by combining the results of dual-luciferase reporter and $\mathrm{Y} 1 \mathrm{H}$ assays.

Two R2R3-MYB genes, RsPAP2 in this study and RsMYB90 reported previously ${ }^{26}$, were isolated from the same red-skinned radish genotype, "NAU-067" (NAU$\mathrm{YH})$. In recent years, R2R3-MYB TFs have been proven to be important participants that regulate ATC biosynthesis in several horticultural plants. The SIAN2-like protein combined with SIMYBATV fine-tunes the accumulation of ATCs in tomato fruit. Both MdMYB10 and $M d M Y B 110$ are regulators of ATC biosynthesis in apple. In this study, the RsPAP2 gene exhibited high expression levels in tissues (e.g., root skin, sepals, petals, and anthers) with abundant ATC accumulation. Similarly, the expression level of the RsMYB90 gene was higher in the taproot skin of "NAU-YH" (red-skinned) than that of "NAU-LB" (white skinned). These results indicated that both the RsPAP2 and RsMYB90 genes might be crucial regulatory genes involved in ATC biosynthesis in radish. In this study, RsPAP2 was confirmed to directly regulate the transcript levels of the RsUFGT gene, and the interaction of RsPAP2 and RsTT8 activated the structural genes RsPAL, RsANS, RsCHI, and RsUFGT to promote anthocyanin biosynthesis. Based on the functional analysis of homologous Arabidopsis genes, it could be inferred that RsMYB90 might regulate anthocyanin-related genes, including the $P A L$ gene of the phenylpropane metabolism pathway. Further functional investigation is required to elucidate the precise roles of the RsPAP2 and RsMYB90 genes involved in the regulatory network of skin color formation in radish. This finding of the present study facilitates a deeper understanding of the genetic regulation of red skin color in radish, providing a valuable resource for the genetic improvement of nutritional quality traits in root vegetable crops.

\section{Materials and methods}

\section{Plant materials and resequencing}

A total of 179 cultivated radish genotypes were used in this study. The taproots of these genotypes exhibit a variety of shapes (e.g., circular, elliptic, transverse elliptic, rectangular, narrow rectangular, and pear-shaped) and colors (e.g., white, red, green, purple, and black) (Table S1). Each genotype was planted at the Jiangpu Breeding Station of Nanjing Agricultural University, Nanjing, China. Genomic DNA was extracted from the young leaves of the 179 radish genotypes via the modified $C T A B$ 
method $^{30}$. Paired-end DNA sequencing libraries with insert sizes ranging from 300 to $500 \mathrm{bp}$ were prepared using the Illumina TruSeq Nano DNA Sample Preparation Kit and sequenced on the Illumina HiSeq 2500 platform.

\section{SNP calling and population genetic analysis}

Raw reads were filtered to remove residual adapters and low-quality bases using SAMtools ${ }^{49}$. The filtered FASTQ files were aligned to the radish reference genome database (WK10039, http://radish-genome.org/) ${ }^{34}$ with BurrowsWheeler Aligner software ${ }^{30}$. After alignment, the "rmdup" command in the SAMtools package was used to remove PCR duplicates according to the mapping coordinates. The SNPs were filtered with a coverage depth $\geq 3$, a minor allele frequency $\geq 0.05$, and a missing rate $<0.2$ to obtain high-quality SNPs.

MEGA 6.0 software was used to generate a neighborjoining phylogenetic tree with 1000 bootstrap replicates ${ }^{50}$. The STRUCTURE program was employed to infer population structure with an admixture $\operatorname{model}^{29}$. The simulations were run with a burn-in of 100,000 iterations and a run length of $10^{6}$ iterations from $K=2-7$. GCTA software (http://cnsgenomics.com/software/gcta/) was employed to perform PCA of individual genotypes. Haploview software was used to assess the LD coefficient $\left(r^{2}\right)$ between pairwise SNPs across the radish genome ${ }^{51}$.

\section{GWAS analysis and candidate gene identification}

The association analysis was performed with the Genome-wide Efficient Mixed-Model Association program based on the SNP data obtained through resequencing ${ }^{52}$. The significance threshold was estimated as $-\log _{10}(P)=7.0$ to identify significantly associated SNPs, which was between the Bonferroni correction value of this study $\left(-\log _{10}(P)=7.4, \quad \alpha=0.05\right)$ and the threshold $\left(-\log _{10}(P)=6.0, \quad \alpha=0.05\right)$ applied in other similar studies $^{14,29}$.

The physical positions of significantly associated SNPs $\left(-\log _{10} P>7.0\right)$ for target traits were used to identify potential candidate genes in the radish genome ${ }^{34}$. The interval for candidate gene identification was $\pm 10 \mathrm{~kb}$ around the SNPs associated with the target traits. The Haploview package was employed to estimate the candidate regions by pairwise LD correlation ${ }^{53}$.

\section{Gene expression analysis}

A total of nine genotypes with different root sizes, shapes, and colors were selected for RNA extraction, which included "NAU-026", "NAU-062", "NAU-067", "NAU-069", "NAU-074", "NAU-112", "NAU-118", "NAU136", and "NAU-152". Using an RNAprep Pure Plant Kit (Tiangen, China), total RNA was isolated from different tissues of the following two groups: the first comprised the root skin and flesh of nine genotypes, and the second comprised the root skin, leaf vein, stem, sepal, petal and anther tissues of two genotypes with red skin and white skin ("NAU-067" and "NAU-069"). The results for the two groups were employed to examine the expression pattern of the candidate RsPAP2 gene.

For RT-qPCR analysis, first-strand cDNA was synthesized from total RNA using the PrimeScript ${ }^{\mathrm{TM}} \mathrm{RT}$ reagent kit with gDNA Eraser (Takara, Dalian, China). Gene expression signatures were generated from three biological replicates in three parallel experiments employing an RT-qPCR approach in a LightCycler ${ }^{\circledR} 480$ System (Roche, Mannheim, Germany) according to the manufacturer's instructions. The relative expression levels were normalized to those of the Actin gene for radish and the elongation factor 1 alpha $(E F 1 \alpha)$ gene for Arabidopsis as internal references and calculated by the $2^{-\Delta \Delta \mathrm{C}_{\mathrm{T}}}$ method $^{54}$. All primers used for RT-qPCR analysis are listed in Table S4.

\section{Sequence analysis}

The protein sequences of the published radish MYBs were downloaded from three previously published radish genome datasets ${ }^{34,35,55}$ and the NCBI database (https:// www.ncbi.nlm.nih.gov/). Related protein sequences of Arabidopsis were obtained from the TAIR database (https://www.arabidopsis.org/). Clustal X and MEGA 6.0 software were used for multiple sequence alignment and phylogenetic analysis, respectively ${ }^{49,56}$.

Conserved cis-acting regulatory elements located in promoter regions were scanned by using PlantCARE (http://bioinformatics.psb.ugent.be/webtools/plantcare/ $\mathrm{html} /$ ), combined with previous reports ${ }^{11}$.

\section{Overexpression vector construction and Arabidopsis transformation}

The full-length coding sequence (CDS) of RsPAP2 was isolated from the red radish genotype "NAU-067" and cloned into the pCAMBIA-2301 vector containing the CaMV35S promoter and the GUS reporter gene using the traditional digestion and ligation method with restriction endonucleases (BamH I and Kpn I) and T4 ligase (Takara, Dalian, China). The recombinant vector was introduced into Agrobacterium tumefaciens strain GV3101 (WeiDi, Shanghai, China). The genetic transformation of Arabidopsis was conducted using the floral-dip method ${ }^{42}$. Transgenic Arabidopsis plants carrying the RsPAP2 gene were identified by selection on halfstrength Murashige and Skoog agar plates containing $100 \mathrm{mg} / \mathrm{L}$ kanamycin, along with assays for $\beta$-glucuronidase activity and RT-qPCR amplification. All primers used for vector construction are shown in Table S4. The leaves and roots of the transgenic Arabidopsis lines were sampled for the measurement of total ATC contents and RT-qPCR analysis. 


\section{Induction of anthocyanins in transiently transformed radish cotyledons}

The full coding region of RsPAP2 was cloned into the multiple cloning sites of the pGreenII 62-SK vectors. The recombinant plasmid was transformed into Agrobacterium strain GV3101 (pSoup) using the freeze-thaw method. The Agrobacterium strain containing RsPAP2 was infiltrated into the abaxial cotyledon surface of the red-skinned radish genotype "NAU-067"57. Cotyledons transformed with pGreenII 62-SK containing a noncoding sequence were used as the control. The phenotype of the cotyledons was monitored and photographed at $9 \mathrm{~d}$ postinfiltration. The infiltrated cotyledon samples were collected for the determination of total ATC contents and RT-qPCR analysis. The primers used for vector construction are shown in Table S4.

\section{Anthocyanin analysis}

A reported methanol- $\mathrm{HCl}$ method with minor modification was used to extract total $\mathrm{ATC}^{9}$. Approximately $0.1 \mathrm{~g}$ of a sample was soaked and incubated for $12 \mathrm{~h}$ in a tube containing $5 \mathrm{~mL}$ of a methanol and $0.1 \%(\mathrm{v} / \mathrm{v}) \mathrm{HCl}$ solution in the dark at room temperature. The ATC concentration was measured and calculated as described by Li et al. ${ }^{58}$. All samples were measured in triplicate with three independent biological replicates.

\section{Dual-luciferase assay of transiently transformed tobacco leaves}

The full-length CDS of RsTT8 was isolated from the red radish genotype "NAU-067" and cloned into the multiple cloning sites of the pGreenII 62-SK vectors. Segmented promoters of ATC biosynthetic genes containing putative MRE and BRE elements were inserted into the cloning site of pGreenII 0800-LUC. All constructs were transformed into Agrobacterium strain GV3101 (pSoup). Agrobacterium culture mixtures of TFs (RsPAP2, RsPAP2 and RsTT8) and promoters (10:1) were infiltrated into 4-weekold tobacco (Nicotiana benthamiana) leaves by using needleless syringes. The infiltration, transient expression analysis, and enzyme activity quantification of Firefly luciferase (LUC) and Renilla luciferase (REN) were conducted. Three days after infiltration, LUC and REN activities were analyzed using a Dual-Luciferase Reporter Assay System (Promega, Wisconsin, USA). Finally, TFpromoter interactions were measured as the ratio of LUC to REN in three independent experiments with at least three biological replicates for each assay. The primers used for the dual-luciferase assay are shown in Table S4.

\section{$\mathrm{Y} 1 \mathrm{H}$ assay}

$\mathrm{Y} 1 \mathrm{H}$ assays were performed using the Matchmaker Gold Yeast One-Hybrid System Kit (Clontech, Palo Alto, USA) to evaluate the binding of RsPAP2 to the MRE and BRE elements in ATC-related gene promoters. These amplified promoter sequences were cloned into the pAbAi vector to generate the bait constructs. The coding region of RsPAP2 was fused in frame with the GAL4 activation domain (AD) in the pGADT7 $\mathrm{AD}$ vector to generate the prey vector (pGADT7-RsPAP2). Bait vectors linearized by BstB I were transformed into the Y1HGold yeast strain to screen the minimal inhibitory concentrations of aureobasidin A (AbA) (Solarbio, Beijing, China). Then, pGADT7-RsPAP2 was introduced into the Y1HGold yeast strain on media lacking Leu (SD/-Leu) supplemented with optimal AbA (100 and $350 \mathrm{ng} / \mathrm{mL}$ ). Empty pGADT7 with the corresponding recombinant pABAi was used as a negative control. The primers used for the $\mathrm{Y} 1 \mathrm{H}$ assay are shown in Table S4.

\section{Acknowledgements}

This work was supported by grants from the Jiangsu Agricultural S\&T Innovation Fund [CX(20)3144], the National Key Technology R\&D Program of China (2017YFD0101806/03), the Fundamental Research Funds for the Central Universities (KYZZ201910), the Jiangsu Agricultural Industry Technology System (JATS2020), and the Priority Academic Program Development of Jiangsu Higher Education Institutions (PAPD).

\section{Author contributions}

L.F. performed the data analysis and wrote the paper. Y.W. and M.T. contributed powerful analytical tools; L.F., J.Y., C.L., J.D., and X.Z. planted the population, scored plant phenotypes, and conducted the validation of experiments; L.L., L.X., and Y.W. helped with the revision of the paper; L.L. and L.F. conceived and designed the research. All authors read and approved the final paper.

Data availability

All raw sequences of the 179 radish genotypes have been deposited in the Sequence Read Archive of the National Center for Biotechnology Information under BioProject number PRJNA634208. The coding sequence of the RSPAP2 gene from NAU-067 has been deposited in GenBank under accession number MT459822.

Conflict of interest

The authors declare that they have no conflict of interest.

Supplementary Information accompanies this paper at (https://doi.org/ 10.1038/s41438-020-00385-y).

Received: 12 March 2020 Revised: 24 June 2020 Accepted: 19 July 2020 Published online: 24 September 2020

\section{References}

1. Naing, A. H. \& Kim, C. K. Roles of R2R3-MYB transcription factors in transcriptional regulation of anthocyanin biosynthesis in horticultural plants. Plant Mol. Biol. 98, 1-18 (2018).

2. Giusti, M. M. N. \& Wrolstad, R. E. Characterization of red radish anthocyanins. J. Food Sci. 61, 322-326 (1996).

3. Wang, L. et al. Regulation of POD activity by pelargonidin during vegetative growth in radish (Raphanus sativus L.). Sci. Hortic. 174, 105-111 (2014).

4. Sun, C. et al. A transcriptional network promotes anthocyanin biosynthesis in tomato flesh. Mol. Plant 13, 42-58 (2020).

5. Schaart, J. G. et al. Identification and characterization of MYB-bHLH-WD40 regulatory complexes controlling proanthocyanidin biosynthesis in strawberry (Fragaria x ananassa) fruits. N. Phytol. 197, 454-467 (2012).

6. Liu, C. et al. Characterization of a citrus R2R3-MYB transcription factor that regulates the flavonol and hydroxycinnamic acid biosynthesis. Sci. Rep. 6 , 25352 (2016).

7. Huang, Y. F. et al. A negative MYB regulator of proanthocyanidin accumulation, identified through expression quantitative locus mapping in the grape berry. N. Phytol. 201, 795-809 (2014). 
8. Liu, Y. et al. Anthocyanin biosynthesis and degradation mechanisms in Solanaceous vegetables: a review. Front. Chem. 6, 52 (2018).

9. Wang, N. et al. The proanthocyanidin-specific transcription factor MdMYBPA1 initiates anthocyanin synthesis under low-temperature conditions in redfleshed apples. Plant J. 96, 39-55 (2018).

10. Wang, X. F. et al. The nitrate-responsive protein MdBT2 regulates anthocyanin biosynthesis by interacting with the MdMYB1 transcription factor. Plant Physiol. 178, 890-906 (2018)

11. Zhu, Z. et al. Characterization of the cis elements in the proximal promoter regions of the anthocyanin pathway genes reveals a common regulatory logic that governs pathway regulation. J. Exp. Bot. 66, 3775-3789 (2015).

12. Teng, S., Keurentjes, J., Bentsink, L., Koornnee, M. \& Smeekens, S. Sucrosespecific induction of anthocyanin biosynthesis in Arabidopsis requires the MYB75/PAP1 gene. Plant Physiol. 139, 1840-1852 (2005).

13. Gonzale, A., Zhao, M., Leavitt, J. \& Lloyd, A. M. Regulation of the anthocyanin biosynthetic pathway by the TTG1/bHLH/Myb transcriptional complex in Arabidopsis seedlings. Plant J. 53, 814-827 (2008).

14. Bac-Molenaar, J. A., Fradin, E. F., Rienstra, J. A., Vreugdenhil, D. \& Keurentjes, J. J. GWA mapping of anthocyanin accumulation reveals balancing selection of MYB90 in Arabidopsis thaliana. PLoS ONE 10, e0143212 (2015).

15. Espley, R. V. et al. Red colouration in apple fruit is due to the activity of the MYB transcription factor, MdMYB10. Plant J. 49, 414-427 (2007).

16. An, J. P. et al. An apple MYB transcription factor regulates cold tolerance and anthocyanin accumulation and undergoes MIEL1-mediated degradation. Plant Biotechnol. J. 18, 337-353 (2020).

17. Feng, S., Wang, Y., Yang, S., Xu, Y. \& Chen, X. Anthocyanin biosynthesis in pears is regulated by a R2R3-MYB transcription factor PyMYB10. Planta 232, 245-255 (2010)

18. Yao, G. et al. Map-based cloning of the pear gene MYB114 identifies an interaction with other transcription factors to coordinately regulate fruit anthocyanin biosynthesis. Plant J. 92, 437-451 (2017).

19. Liu, X. F. et al. The role of MrbHLH1 and MrMYB1 in regulating anthocyanin biosynthetic genes in tobacco and Chinese bayberry (Myrica rubra) during anthocyanin biosynthesis. Plant Cell Tissue Organ. 115, 285-298 (2013).

20. Liu, Y. et al. Functional diversification of the potato R2R3 MYB anthocyanin activators AN1, MYBA1, and MYB113 and their interaction with basic helixloop-helix cofactors. J. Exp. Bot. 67, 2159-2176 (2016).

21. M'mbone, M. E. et al. Coordinated regulation of anthocyanin biosynthesis genes confers varied phenotypic and spatial-temporal anthocyanin accumulation in radish (Raphanus sativus L.). Front. Plant Sci. 8, 1243 (2017).

22. Lim, S. H. et al. Activation of anthocyanin biosynthesis by expression of the radish R2R3-MYB transcription factor gene RsMYB1. Plant Cell Rep. 35, 641-653 (2016).

23. Yi, G. et al. MYB1 transcription factor is a candidate responsible for red root skin in radish (Raphanus sativus L.). PLoS ONE 13, e0204241 (2018).

24. Liu, T. et al. Combined QTL-Seq and traditional linkage analysis to identify candidate genes for purple skin of radish fleshy taproots. Front. Genet. 10, 808 (2019).

25. Lai, B. et al. Differential anthocyanin accumulation in radish taproot: importance of RsMYB1 gene structure. Plant Cell Rep. 39, 217-226 (2020).

26. Luo, X. et al. An ultra-high-density genetic map provides insights into genome synteny, recombination landscape and taproot skin colour in radish (Raphanus sativus L.). Plant Biotechnol. J. 1, 274-286 (2020).

27. Wang, Q., Wang, Y., Sun, H., Sun, L. \& Zhang, L. Transposon-induced methylation of the RsMYB1 promoter disturbs anthocyanin accumulation in redfleshed radish. J. Exp. Bot. 71, 2537-2550 (2020).

28. Su, J. et al. Genome-wide association study identifies favorable SNP alleles and candidate genes for waterlogging tolerance in chrysanthemums. Hortic. Res. 6, 21 (2019).

29. Zhang, L. et al. RNA sequencing provides insights into the evolution of lettuce and the regulation of flavonoid biosynthesis. Nat. Commun. 8, 2264 (2017).

30. Cao, K. et al. Genome-wide association study of 12 agronomic traits in peach. Nat. Commun. 7, 13246 (2016).

31. Cardoso, S., Lau, W., Eiras, D. J., Fevereiro, P. \& Maniatis, N. A candidate-gene association study for berry colour and anthocyanin content in Vitis vinifera $\mathrm{L}$. PLOS ONE 7, e46021 (2012).

32. Cericola, F. et al. Linkage disequilibrium and genome-wide association analysis for anthocyanin pigmentation and fruit color in eggplant. BMC Genom. 15, 896 (2014).
33. Schulz, D. F. et al. Genome-wide association analysis of the anthocyanin and carotenoid contents of rose petals. Front. Plant Sci. 7, 1798 (2016).

34. Jeong, Y. M. et al. Elucidating the triplicated ancestral genome structure of radish based on chromosome-level comparison with the Brassica genomes. Theor. Appl. Genet. 129, 1357-1372 (2016).

35. Mitsui, Y. et al. The radish genome and comprehensive gene expression profile of tuberous root formation and development. Sci. Rep. 5, 10835 (2015).

36. Kim, N. et al. Identification of candidate domestication regions in the radish genome based on high-depth resequencing analysis of 17 genotypes. Theor. Appl. Genet. 129, 1797-1814 (2016).

37. Yang, L. et al. Response of plant secondary metabolites to environmental factors. Molecules 23, 762 (2018).

38. Flint-Garcia, S. A., Thornsberry, J. M. \& Buckler, E. S. Structure of linkage disequilibrium in plants. Annu. Rev. Plant Biol. 54, 357-374 (2003).

39. Yan, S. et al. Anthocyanin Fruit encodes an R2R3-MYB transcription factor, SIAN2-like, activating the transcription of SIMYBATV to fine-tune anthocyanin content in tomato fruit. N. Phytol. 5, 2048-2063 (2020).

40. Butelli, E. et al. Retrotransposons control fruit-specific, cold-dependent accumulation of anthocyanins in blood oranges. Plant Cell 24, 1242-1255 (2012).

41. Chagné, D. et al. An ancient duplication of apple MYB transcription factors is responsible for novel red fruit-flesh phenotypes. Plant Physiol. 161, 225-239 (2013).

42. Fu, W. et al. Production of red-flowered oilseed rape via the ectopic expression of Orychophragmus violaceus OvPAP2. Plant Biotechnol. J. 16, 367-380 (2018).

43. Anwar, M. et al. Ectopic overexpression of a novel R2R3-MYB, NtMYB2 from Chinese Narcissus represses anthocyanin biosynthesis in tobacco. Molecules 23, 781-799 (2018).

44. Feng, $K$. et al. AgMYB2 transcription factor is involved in the regulation of anthocyanin biosynthesis in purple celery (Apium graveolens L.). Planta 248, 1249-1261 (2018).

45. Bai, S. et al. Transcriptome analysis of bagging-treated red Chinese sand pear peels reveals light-responsive pathway functions in anthocyanin accumulation. Sci. Rep. 7, 63 (2017).

46. Matus, J. T. et al. A group of grapevine MYBA transcription factors located in chromosome 14 control anthocyanin synthesis in vegetative organs with different specificities compared with the berry color locus. Plant J. 91, 220-236 (2017).

47. Zou, B. et al. Calmodulin-binding protein CBP60g functions as a negative regulator in Arabidopsis anthocyanin accumulation. PLOS ONE 12, e0173129 (2017).

48. Borevitz, J. O., Xia, Y., Blount, J., Dixon, R. T. \& Lamb, C. Activation tagging identifies a conserved MYB regulator of phenylpropanoid biosynthesis. Plant Cell 12, 2383-2393 (2000).

49. Su, J. et al. Genome-wide association study identified genetic variations and candidate genes for plant architecture component traits in Chinese upland cotton. Theor. Appl. Genet. 131, 1299-1314 (2018).

50. Tamura, K., Stecher, G., Peterson, D., Filipski, A. \& Kumar, S. MEGA6: molecular evolutionary genetics analysis version 6.0. Mol. Biol. Evol. 30, 2725-2729 (2013).

51. Crowell, $\mathrm{S}$. et al. Genome-wide association and high-resolution phenotyping link Oryza sativa panicle traits to numerous trait-specific QTL clusters. Nat. Commun. 7, 10527 (2016).

52. Zhou, X. \& Stephens, M. Genome-wide efficient mixed-model analysis for association studies. Nat. Genet. 44, 821-824 (2012).

53. Gautam, K. A. et al. Functional polymorphisms in the IL6 gene promoter and the risk of urinary bladder cancer in India. Cytokine 77, 152-156 (2016).

54. $\mathrm{Xu}, \mathrm{Y}$. et al. Evaluation of reference genes for gene expression studies in radish (Raphanus sativus L.) using quantitative real-time PCR. Biochem. Biophys. Res. Commun. 424, 398-403 (2012).

55. Zhang, $X$. et al. A de novo genome of a Chinese radish cultivar. Hortic. Plant $J$. 1, 155-164 (2015).

56. Larkin, M. A. et al. Clustal W and Clustal X version 2.0. Bioinformatics 23 2947-2948 (2007)

57. Lim, S. H. et al. Use of an anthocyanin production phenotype as a visible selection marker system in transgenic tobacco plant. Plant Biotechnol. Rep. 6, 203-211 (2012).

58. Li, Y. Y. et al. MdCOP1 ubiquitin E3 ligases interact with MdMYB1 to regulate light-induced anthocyanin biosynthesis and red fruit coloration in apple. Plant Physiol. 160, 1011-1022 (2012). 